\title{
NOTE ON AN INTEGRAL OF BIERENS DE HAAN
}

\author{
THOMAS JAMES HIGGINS
}

In connection with certain potential problems, the author has recently had occasion to evaluate integrals of the form

$$
I_{n}=\int_{0}^{\infty} \frac{\epsilon^{-p x}}{x^{n+1}} \sin q_{0} x \cdot \sin q_{1} x \cdot \sin q_{2} x \cdots \sin q_{n} x d x .
$$

In Table 371 of Bierens de Haan's Nouvelles Tables d'Intégrales Définies a rule is given for evaluation of this integral. An attempt to use it led to finding that it was incorrect.

This integral was originally considered by de Haan in a paper published in Dutch in the rather inaccessible Archief, Wiskundig Genootschap onder de Zinspreuk, part 1, 1856-1859, pp. 288-315. Reference to this article revealed that while de Haan did not give a specific rule for the above integral, he did discuss it in general. Evidently, in the compilation of these tables in French a garbled version of this discussion was inserted. As integrals of this form occur in several potential problems of current interest, ${ }^{1}$ it is thought worthwhile to point out this error in these universally used tables and to give the following rule, ascertained partly from de Haan's discussion and partly from direct inspection of a number of specific cases given in the same article. The correct formula is:

$$
I=\frac{1}{n ! 2^{n}} \sum_{j=1}^{j=2^{n}}(-1)^{r_{j}}\left[T_{j} \arctan \frac{k_{j}}{p}-L_{j} \log \left(k_{j}^{2}+p^{2}\right)^{1 / 2}\right],
$$

where

(a) $k_{j}$ is any one of the $2^{n}$ possible sums obtainable from $\left(q_{0} \pm q_{1} \pm q_{2} \pm \cdots \pm q_{n}\right)$ by selection of the + and - signs;

(b) $r_{j}$ is the number of negative signs in $k_{j}$;

(c) $T_{j}$ is the sum of the 1 st, $3 \mathrm{rd}, 5 \mathrm{th}, \ldots$ terms of

$$
\begin{aligned}
k_{j}^{n}+C_{n, n-1} p k_{j}^{n-1} & -C_{n, n-2} p^{2} k_{j}^{n-2}-C_{n, n-3} p^{3} k_{j}^{n-3} \\
& +C_{n, n-4} p^{4} k_{j}^{n-4}+C_{n, n-5} p^{5} k_{j}^{n-5}-C_{n, n-6} p^{6} k_{j}^{n-6}-\cdots p^{n}
\end{aligned}
$$

1 The author encountered these integrals in seeking to determine the vector potential of two parallel, infinitely long, tubular rectangular conductors carrying currents in opposite directions. In general, they arise in two dimensional problems, where it is necessary to obtain a potential satisfying Poisson's equation over certain rectangular areas and Laplace's equation over the remainder of space. 
(d) $L_{j}$ is the sum of the remaining terms.

As an illustration we evaluate the integral

Now

$$
I_{3}=\int_{0}^{\infty} \frac{\epsilon^{-p x}}{x^{3}} \sin ^{2} a x \cdot \sin b x d x
$$

$$
\begin{array}{ll}
k_{1}=a+a+b=2 a+b ; & (-1)^{r_{1}}=1 ; \\
k_{2}=a+a-b=2 a-b ; & (-1)^{r_{2}}=-1 ; \\
k_{3}=a-a+b=b ; & (-1)^{r_{3}}=-1 ; \\
k_{4}=a-a-b=-b ; & (-1)^{r_{4}}=1 ;
\end{array}
$$

and from

$$
k_{j}^{2}+C_{2,1} p k_{j}-p^{2}
$$

we have

$$
T_{j}=k_{j}^{2}-p^{2}, \quad L_{j}=2 p k_{j} .
$$

Hence

$$
I_{3}=\frac{1}{2^{2} 2 !} \sum_{j=1}^{j=4}(-1)^{r_{j}}\left[\left(k_{j}^{2}-p^{2}\right) \arctan \frac{k_{j}}{p}-2 p k_{j} \log \left(k_{j}^{2}+p^{2}\right)^{1 / 2}\right] .
$$

This confirms the integral \#2, Table 370 .

Purdue University 